\title{
A case of entero-colic fistula formation resulting from stent migration inserted for obstructing left colonic tumour
}

\author{
Atif Alvi • James P. Pitt
}

Accepted: 27 November 2008 / Published online: 16 December 2008

(C) Springer-Verlag 2008

\section{Dear Editor:}

Management of malignant obstruction of large bowel is associated with a number of challenges. Favourable outcomes have been reported with the use of SEMS both for cancer palliation and treatment of acute obstruction as a "bridge to surgery". Colonic stent migration can occur in 3$22 \%$ of cases. Stenosis from extrinsic compression and laser pre-treatment or chemotherapy may be factors precipitating stent migration. We experienced one case, however, of a patient in whom a SEMS implanted for obstructing distal descending colon tumour resulted in an entero-colic fistula formation due migration. We describe this complication and discuss the possible causes of fistula formation.

An 85-year-old man was admitted with a closed loop large bowel obstruction. A CT scan confirmed an obstructing stricture in the distal descending colon. His past medical history included MI and CABG in 2000, a right hemicolectomy for Dukes B adenocarcinoma in 2001. Laparotomy for a defunctioning procedure was planned but just prior to that he suffered a non-ST elevating myocardial infarction. Therefore, a self-expanding metallic stent was placed successfully in the descending colon stricture.

A week later, he represented with acute large bowel obstruction due to blocked stent. Laparotomy revealed an obstructing tumour. A Hartmann's procedure was performed with removal of left colon and with an assumption

A. Alvi $(\varangle) \cdot$ J. P. Pitt

Department of Colorectal Surgery, The Ipswich Hospital,

Heath Road,

Ipswich IP4 5PD, UK

e-mail: atifalvi@yahoo.co.uk

that the stent would be in the resected specimen, as palpation of the remaining colon did not identify the stent. Histology confirmed Dukes B adenocarcinoma.

He was readmitted 2 weeks later with bleeding from the stoma but settled quickly and was sent home once again. Six weeks after the Hartmann's procedure, he was admitted with vomiting, lower abdominal pain and loose stools for 2 weeks. A plain abdominal radiograph revealed the stent present in the colon. At laparotomy, the stent had eroded through the colonic wall $20 \mathrm{~cm}$ proximal to the stoma and fistulated into two loops of jejunum approximately $30 \mathrm{~cm}$ from the duodeno-jejunal flexure. The fistula was taken down, small bowel and colon were repaired primarily and a reversal of Hartmann's was performed with a covering defunctioning loop ileostomy. Post-operatively, the patient suffered from pneumonia and with gradual deterioration, died with respiratory failure.

Acute colorectal obstruction is traditionally approached with segmental colectomy and diverting colostomy. However, up to $25-40 \%$ of patients never undergo colostomy closure, and colostomy closure itself carries a $7 \%$ mortality rate. Tumour ingrowth and overgrowth resulting in the recurrence of obstructive symptoms is the main long-term complication of SEMS placement. The use of covered stents is believed to reduce tumour ingrowth; however, it may lead to an increased stent migration rate. Wada et al. reported a case of stent perforation and ileosigmoid fistula formation in a patient with Crohn's disease. Suzuki et al. showed seven of 36 patients suffered from stent migration with one of 36 resulting in fistula formation. Sofiya et al., in her 5-year literature review, showed no fistula formation and only 3\% stent migration. Migration and fistula formation is a rare but recognised complication of colonic stent placement for obstructed colon. 